\section{3,9 millones de muertes en el mundo han sido prevenidas por la práctica regular de actividad física: ¿̨cuál es la realidad en Chile?}

\section{Regular physical activity in Chile: Preventing premature mortality}

\section{Señor Editor:}

Existe suficiente evidencia científica que vincula la práctica regular de actividad física $(\mathrm{AF})$ con un menor riesgo de desarrollar enfermedades cardiovasculares, tipos de cáncer y muertes prematuras, sin embargo, $27,5 \%$ de la población adulta a nivel mundial $(23,4 \%$ y $31,7 \%$ en hombres y mujeres, respectivamente $)^{1}$, no cumple con las actuales recomendaciones de AF para la salud, las cuales sugieren realizar al menos $150 \mathrm{~min}$ de AF moderada o 75 min de AF vigorosa o una combinación equivalente de ambas por semana.

Una de las estrategias utilizadas frecuentemente por salubristas públicos para comunicar el impacto que tiene la AF en la salud, es a través de la estimación del riesgo de desarrollar enfermedades o mortalidad asociada a ser físicamente inactivo. Esta implica destacar los efectos adversos asociados al factor de exposición, en este caso AF, con el fin de promover cambios en los estilos de vida de la población. Sin embargo, otra forma de transmitir los efectos de la AF en la salud de las personas, es mediante la adopción de un escenario que destaque los beneficios asociados a ser físicamente activo. Dicho escenario se enfoca en las personas que sí cumplen las recomendaciones y obtienen beneficios en la salud, versus aquellas que no han cumplido con esas recomendaciones. Esta herramienta epidemiológica llamada fracción prevenible de la población o PFP (del inglés Prevented Fraction of the Population), permite estimar la cantidad de casos o muertes que han sido prevenidas debido a un factor de exposición protector, que en este caso es el cumplimiento de las recomendaciones de AF.

Un estudio reciente publicado en la prestigiosa revista Lancet Global Health², utilizó esta herramienta epidemiológica para estimar las muertes prematuras, es decir, muertes entre los 40 y 74 años, que han sido prevenidas en el mundo durante el año 2019, producto del cumplimiento de las recomendaciones de $\mathrm{AF}^{2}$. Esta investigación que incluyó datos de AF de 1,9 millones de personas pertenecientes a 168 países, entre ellos Chile, estimó que 3,9 millones de potenciales muertes prematuras a nivel mundial fueron prevenidas por AF, equivalentes a $15,0 \%$ del total de muertes prematuras durante el año 2019 a nivel global ${ }^{2}$. De esta cifra, 1.5 millones fueron mujeres $(14,1 \%)$ y 2,4 millones fueron hombres $(16,0 \%)$. Al analizar por regiones del mundo, África presentó la mayor cantidad de muertes prematuras prevenidas por $\mathrm{AF}$ (487.200 muertes), mientras que América, reportó la menor cantidad (349.300 muertes). También se encontraron claras diferencias en la cantidad de muertes prematuras prevenidas por AF en los países, según el ingreso económico de estos. De esta manera, en primer lugar surgen los países de nivel económico bajo en los cuales se previnieron $17,9 \%$ del total de muertes prematuras ocurridas en estos países (equivalente a 269.300 muertes), después los países de nivel medio-bajo con $16,5 \%(n=1.529 .900)$, en tercer lugar se ubican aquellos de nivel económico alto con $14,1 \%(n=509.300)$, y en último lugar países de ingreso económico medio-alto con $14,0 \%$ muertes prevenidas $(\mathrm{n}=1.621 .000)^{2}$.

$\mathrm{Si}$ analizamos el contexto latinoamericano, del total de fallecimientos registrados durante el año 2019, los países con la mayor proporción de muertes prematuras prevenidas por AF fueron Uruguay $(16,4 \%)$, Chile $(15,4 \%)$ y Ecuador $(15,3 \%)$, mientras que Brasil $(10,8 \%)$, Costa Rica $(11,1 \%)$ y Argentina $(12,0 \%)$ presentaron el menor porcentaje (Figura 1). Este orden en los países se mantuvo al estratificar los resultados por sexo ${ }^{2}$. Sin embargo, si consideramos el número de muertes absolutas prevenidas Brasil y México lideran el ranking con 71.600 y 41.300 muertes prematuras prevenidas por AF, respectivamente. Esta gran diferencia en muertes absolutas se atribuye al tamaño de la población total de estos países (Figura 1). 
En Chile, la proporción de la población que es físicamente activa, en base a las recomendaciones de AF, correspondió a 73,4\% (71,4\% y 75,6\% en mujeres y hombres, respectivamente) $)^{2}$. Estos antecedentes representan la segunda prevalencia más alta de cumplimiento de las recomendaciones de $\mathrm{AF}$ en Latinoamérica después de Uruguay $(77,6 \%)$. En este sentido, solo $65,2 \%$ de la población que murió prematuramente en Chile durante el año 2019 era físicamente activa $(62,9 \%$ y $67,8 \%$ para mujeres y hombres, respectivamente). En base a estas estimaciones, el número de muertes prevenidas

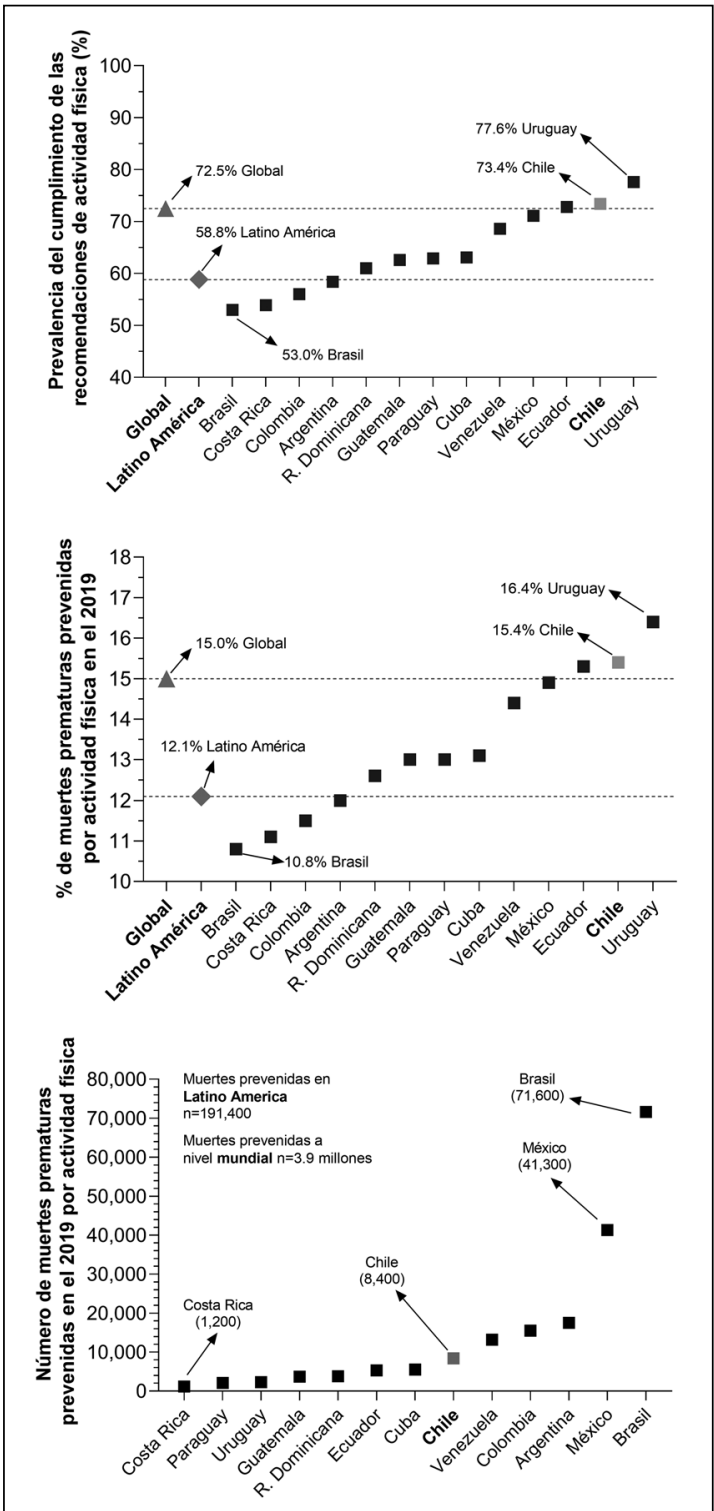

Figura 1. Muertes prematuras prevenidas por actividad física en el año 2019. Datos adaptados de Strain et al. ${ }^{2}$. en Chile durante el año 2019, debido al cumplimiento de las recomendaciones de AF correspondió a 8.400, equivalente a $15,4 \%$ de todas las muertes prematuras ocurridas en el país (Figura 1). De estas, $3.400(15,0 \%)$ y $5.000(15,9 \%)$ fueron prevenidas en mujeres y hombres, respectivamente (Figura 1$)^{2}$.

Si bien Chile presenta una de las prevalencias más altas de cumplimiento de las recomendaciones de $\mathrm{AF}$ en Latinoamérica, ubicándose como el segundo país de la región con la mayor proporción de muertes prevenidas por $\mathrm{AF}$, aún existe un $26,6 \%$ de la población chilena que no cumple con dichas recomendaciones. Por lo cual, es necesario continuar fomentando la práctica regular de AF a través del diseño y ejecución de políticas y programas que impacten significativamente en todos los sectores de la población.

\section{Carlos Celis-Morales ${ }^{1,2}$, Carlos Salas-Bravo ${ }^{3}$,} Carlos Matus-Castillo ${ }^{4}$

${ }^{1}$ Institute of Cardiovascular and Medical Sciences, University of Glasgow, Glasgow, United Kingdom.

${ }^{2}$ Laboratorio de Rendimiento Humano, Grupo de Estudio en Educación, Actividad Física y Salud (GEEAFyS), Universidad Católica del Maule. Talca, Chile.

${ }^{3}$ Departamento de Educación Física, Facultad de Educación, Universidad de Concepción. Concepción, Chile.

${ }^{4}$ Departamento de Ciencias del Deporte $y$ Acondicionamiento Físico. Universidad Católica de la Santísima Concepción. Concepción, Chile.

\section{Referencias}

1. Guthold R, Stevens GA, Riley LM, Bull FC. Worldwide trends in insufficient physical activity from 2001 to 2016: a pooled analysis of 358 population-based surveys with 1.9 million participants. Lancet Glob Health 2018; 6 (10): e1077-86. Available from: https://doi.org/10.1016/S2214109X(18)30357-7.

2. Strain T, Brage S, Sharp SJ, Richards J, Tainio M, Ding D, et al. Use of the prevented fraction for the population to determine deaths averted by existing prevalence of physical activity: a descriptive study. Lancet Glob Health 2020; 8 (7): e920-30. Available from: https://doi.org/10.1016/S2214109X(20)30211-4.

Correspondencia a:

Dr. Carlos Celis-Morales

BHF Glasgow Cardiovascular Research Centre.Institute of

Cardiovascular and Medical Sciences. University of Glasgow. Glasgow, G12 8TA, United Kingdom.

Carlos.Celis@glasgow.ac.uk 